\title{
Die französische Geschichtsschreibung am Anfang des 21. Jahrhunderts - einige Einblicke
}

\section{Nicolas Offenstadt}

Traducteur : Niels F. May et Volker Zimmermann

\section{OpenEdition}

Journals

Édition électronique

URL : http://journals.openedition.org/ifha/7835

DOI : $10.4000 /$ ifha. 7835

ISSN : 2198-8943

Éditeur

IFRA - Institut franco-allemand (sciences historiques et sociales)

Référence électronique

Nicolas Offenstadt, «Die französische Geschichtsschreibung am Anfang des 21. Jahrhunderts einige Einblicke », Revue de l'IFHA [Online], HS | 2014, Online erschienen am: 01 September 2014 aufgerufen am 01 Mai 2019. URL : http://journals.openedition.org/ifha/7835 ; DOI : 10.4000/ifha.7835

Ce document a été généré automatiquement le 1 mai 2019.

(C)IFHA 


\title{
Die französische Geschichtsschreibung am Anfang des 21. Jahrhunderts - einige Einblicke
}

\author{
Nicolas Offenstadt \\ Traduction : Niels F. May et Volker Zimmermann
}

\section{NOTE DE L'ÉDITEUR}

Aus dem Französischen übersetzt von Niels F. May und Volker Zimmermann.

1 Vor nicht allzu langer Zeit, in den Jahren 2005-2006, haben die französischen Historikerinnen und Historiker den öffentlichen Raum erobert oder - wenn man so will sie wurden von ihm erobert (und zwar mit einer ungewöhnlichen Intensität) : Debattiert wurde unter anderem über die Rolle von Geschichte in der Republik, über die mit den verschiedenen Erinnerungskulturen verbundenen Forderungen, darüber, ob Gesetze die Rolle eines Schiedsrichters im Umgang mit der Vergangenheit annehmen können usw. Die Vergangenheit erschien dabei als eine Gegenwart mit großen Spannungen. Die Geschichtsschreibung - gerade in Frankreich - kann deswegen gänzlich nur innerhalb eines größeren Kontexts verstanden werden, der über den Rahmen der akademischen Welt im engeren Sinn hinausgeht. Wir werden auf diesen Aspekt zurückkommen.

Die französische Geschichtsschreibung - wie ihre Pendants in anderen Ländern auch steht zweifellos mehr und mehr unter dem Eindruck einer weltweiten Standardisierung von Einstellungs- und Evaluationskriterien sowie der raschen Verbreitung von Daten und Wissensbeständen. Dieser doppelte Kontext - einerseits die Integration in einen breiten öffentlichen Raum und andererseits die fortschreitende und immer schnellere Internationalisierung - muss bei allen Überlegungen zur aktuellen französischen Geschichtsschreibung berücksichtigt werden. Der zweite Aspekt betrifft nicht die französische Historiographie allein, wir werden ihn deshalb hier nicht gesondert 
behandeln. Der erste hingegen ist ein spezifisches Merkmal der aktuellen französischen Situation und verdient deshalb, einführend näher untersucht zu werden, bevor wir aufzeigen werden, auf welche vielfältige Weise französische Historikerinnen und Historiker heute die von ihnen angelegten Maßstabsebenen variieren und so die Geschichtsschreibung erneuern. Abschließend werden einige Forschungsfelder aufgezeigt, die gegenwärtig besonders dynamisch erscheinen.

\section{Kontext}

Dass der soziale Kontext - auf unterschiedliche Weise - Einfluss auf die akademische Welt und die Orientierungen der Forschung ausübt, ist keine neue Erkenntnis. Vor allem Jubiläumsfeierlichkeiten bieten Verlagen und Geschichtswissenschaft den Anlass zur Veröffentlichung von Werken, die sich auf das jeweils zu feiernde historische Ereignis beziehen. Dabei kann es sich um regelmäßig wiederkehrende Zeitpunkte des Gedenkkalenders wie den 11. November (1918) oder den 8. Mai (1945) handeln, aber auch um außergewöhnlichere Momente aus Anlass von fünfzig- oder hundertjährigen Jubiläen (wie der Französischen Revolution 1989) oder gar Tausendjahrfeiern (wie der KapetingerDynastie 1987 oder der Taufe Chlodwigs I. im Jahr 1996). Besonders augenfällig wird dies im Fall des Ersten Weltkriegs 1914-1918 und den ihm gewidmeten Gedenkveranstaltungen in den Jahren 1964 bis 1968 bzw. 1998 - von der im Jahr 2014 zelebrierten Hundertjahrfeier ganz zu schweigen.

Daneben beeinflussen die heftigsten öffentlichen Debatten oft auch die Auswahl der Themen und Fragen, die Historikerinnen und Historiker - mehr oder weniger explizit, mit mehr oder weniger großem Nachdruck - an die Geschichte stellen. Dies wird auch dadurch begünstigt, dass seit den Dezentralisierungsgesetzen von 1982/1983 Dissertationen und Tagungen ganz oder teilweise von den Conseils régionaux (entspricht in etwa den deutschen Landtagen) und den Conseils généraux (Parlamente der Départements) finanziert werden.

5 Seit einigen Jahrzehnten haben jedoch die Herausforderungen in Bezug auf die verschiedenen »Gedächtnisse« (des Staates und der öffentlichen Körperschaften, aber auch von institutionell verankerten oder informellen Gruppen) in der öffentlichen Debatte an Bedeutung gewonnen. Denn die Vergangenheit ist heute - also zu einer Zeit, da die in den 60er und 70er Jahren heiß diskutierten Zukunftsentwürfe ihre Evidenz verloren haben - selbst eine wichtige »Ressource" geworden. Die großen Meistererzählungen, die diese Konzepte unterfütterten (das nationale Epos, die revolutionäre Emanzipation usw.) und in einem gewissen Austausch auch mit der Geschichtsschreibung standen, haben in der Tat ihre Überzeugungskraft eingebüßt. Viele französische Historikerinnen und Historiker - vor allem jene, die zur Zeitgeschichte arbeiten (aber nicht nur, wie die Polemiken zu den Kreuzzügen oder zu den Katharern zeigen, in deren Kontext auch touristische Überlegungen auf dem Spiel stehen) - spüren zunehmend den Konkurrenzdruck anderer Diskurse, die oft unter dem Terminus Erinnerungskulturen zusammengefasst werden.

6 Es handelt sich dabei um mehr oder weniger gut organisierte Gruppen von Akteuren der Geschichte (wie z. B. die französischen Widerstandskämpfer, deren Zahl heute immer geringer wird), ihre erklärten Erben (z. B. Nachfahren von Sklaven oder von Überlebenden des Völkermords an den Armeniern) oder aber »Erinnerungsaktivisten« ohne direkten Bezug zu den von ihnen verteidigten 
Gegenständen (wie im Fall der Gruppen, die eine Rehabilitierung der im Ersten Weltkrieg hingerichteten Soldaten fordern). Alle diese Akteure sprechen und schreiben öffentlich über die Geschichte und stellen mitunter präzise Forderungen auf zum Platz ihrer eigenen Vergangenheit oder jener, die im nationalen Gedenken oder schulischen Lehrplänen behandelt wird. Hierbei steht einiges auf dem Spiel, denn die Lehrpläne der Primär- und Sekundarstufe werden für das gesamte Land vom Bildungsministerium (Ministère de l'Éducation Nationale) festgelegt. Einige Themen, wie das Massaker an den Algeriern am 17. Oktober 1961 in Paris, werden zunächst außerhalb der akademischen Welt thematisiert, bevor sie von staatlicher Seite aufgegriffen werden (so geschehen durch François Hollande 2012). Diese »Erinnerungs «-Diskurse und die Debatten, die sie hervorrufen, haben viele professionelle Historikerinnen und Historiker bewogen, Stellung zu beziehen, aber sie haben auch Arbeiten hervorgebracht, die von allgemeinerem Interesse sind ${ }^{1}$.

7 Ein ganzes Bündel von grundlegenden Tendenzen hat sich im Jahr 2005 herausgebildet. Anlass war die Verabschiedung des Gesetzes vom 23. Februar »über die Anerkennung seitens der Nation und die nationalen Leistungen zugunsten der repatriierten Franzosen« . Der vierte Paragraph dieses Gesetzes besagte : „Die Lehrpläne erkennen die positive Rolle der französischen Präsenz in den Überseegebieten an, speziell in Nordafrika, und weisen der Geschichte und den Opfern der Kämpfer der französischen Armee, die aus diesen Territorien stammen, den ihr gebührenden Platz zu« ${ }^{2}$. Sehr rasch verurteilten zahlreiche Historikerinnen und Historiker diese erhebliche staatliche Einflussnahme auf die Lehrpläne. Die Debatte gewann dadurch an Tragweite, dass der Historiker Olivier Pétré-Grenouilleau, Spezialist der Geschichte der Sklaverei, von einer Gruppe von engagierten Bürgern aus den DOM-TOM (Territoires d'outre-mer et Départements d'outre-mer = französische Überseegebiete) juristisch verfolgt wurde. Grund war, dass er in einem Zeitungsartikel die Meinung geäußert hatte, man könne den Sklavenhandel nicht als Völkermord bezeichnen (Journal du Dimanche vom 12. Juni 2005). Es war nicht das erste Mal, dass ein Historiker derart angegriffen wurde; die angespannte Situation in Fragen der Geschichte trug jedoch dazu bei, dass sich die Historikerzunft in Solidarität um ihn scharte. Zwischen dem Einschärfungsgesetz für die Geschichtslehrerinnen und -lehrer und dieser Verfolgung vor Gericht wurde jedenfalls in diesen Jahren das Fach »Geschichte« von allen Seiten durch die verschiedenen "Erinnerungskulturen« und die politischen Vertretungen, die sich auf dem Weg der Gesetzgebung zu ihrem Sprachrohr machten, in die Mangel genommen. Weitere Diskussionspunkte kamen zu diesen Fragen noch hinzu. In der Summe, so konstatierte der Historiker Patrick Garcia, stellten diese Diskussionen daher seit dem Lehrplanstreit der frühen achtziger Jahre die wichtigste Debatte all jener dar, die sich mit der Geschichte beschäftigen ${ }^{3}$.

Genau genommen sprachen die Historikerinnen und Historiker in diesen Debatten jedoch nicht mit einer Stimme - man muss sich hier vor Verallgemeinerungen hüten. Viele Diskussionsbeiträge - etwa der Aufruf zur Verteidigung der »Freiheit der Geschichte« von 2005 - konzentrierten sich auf die Ablehnung von Gesetzen, die das kollektive Gedächtnis regulieren sollten (die sogenannten »Erinnerungsgesetze $\aleph^{4}$ ). Für diejenigen, die diese Position unterstützten, kamen die Einmischung der Politik qua Gesetz und der Druck der Erinnerungsgemeinschaften zumindest potentiell einer Beeinträchtigung der Forschung gleich. Pierre Nora schoss über das Ziel hinaus und ging so weit, von einer »Sowjetisierung der Geschichte« zu sprechen (Le Monde vom 27.12.2011). Hinter dieser Ansicht stand kaum verhüllt ein politischer Diskurs über die Einheit der Nation, 
die von den Erinnerungskulturen bedroht sei - im Sinne der Idee, dass die Geschichte (als eine distanzierte und wissenschaftliche Analyse) »zusammenführe«, während die Erinnerungskulturen (als eine subjektive und beschränkte Herangehensweise an die Vergangenheit ) »spalteten« .

Diese Abwehr der »Zunft« gegen das ihr Äußerliche, d.h. gegen die Erinnerungskulturen und "Erinnerungsgesetze«, wird von anderen Historikerinnen und Historikern auch als die »Identitätskrise« einer Berufsgemeinschaft interpretiert, die sich der sozialen Dimension der Vergangenheit zu wenig zugewandt habe (etwa bei Patrick Boucheron oder in Deutschland Valentin Groebner ${ }^{5}$ ). Wieder andere, zu denen ich selbst gehöre, fragen sich, in wessen Namen die Historikerinnen und Historiker eigentlich beanspruchen, die Vergangenheit allein in ihrem Elfenbeinturm zum Heiligtum zu dekretieren, ohne sich um die mitunter so wichtigen sozialen Zusammenhänge und die politischen Ausdrucksformen, die diesen in einer Demokratie zukommen können, zu scheren. Die historische Zunft sollte sich vielmehr als eine Quelle der Erkenntnis für die Debatten begreifen, die außerhalb ihres Feldes stattfinden, und sie nicht von vornherein verurteilen.

Aus einer im engeren Sinne historiographischen Perspektive heraus hat die Gesamtheit dieser Debatten darüber hinaus Arbeiten zur Konstruktion der Nationalgeschichte (mehrere Vorhaben beschäftigen sich so mit einer Neuinterpretation der französischen Geschichte, etwa die im Verlag Belin erscheinende und von Joël Cornette herausgegebene Reihe) und zum Umgang mit der Vergangenheit angeregt. Gleiches gilt auch für eine in verschiedenen Formen neue Geschichte der Kolonien und der Kolonialisierung, die sich ebenfalls mit öffentlichen Diskussionen vermischt ${ }^{6}$.

\section{Variationen der Maßstabsebenen}

11 Einer der markantesten Aspekte der neueren Entwicklungen der Geschichtswissenschaft ist die Auflösung von »Nation« und »Region« als für die historische Monographie gültiger Rahmen bzw. klassischer, für die Dissertation oder die Überblicksdarstellungen verbindlicher Maßstabsebene. Heutzutage hantieren die französischen Historikerinnen und Historiker in ihren Beschreibungen und Erläuterungen mit allen Größenordnungen, von der kleinsten bis zur größten. Das Aufweichen der Definitionsrahmen für Themen liegt sowohl an der räumlichen Reichweite von Forschungsarbeiten (die sich heute mit einer Straße beschäftigen können, aber auch mit den globalen Austauschbeziehungen) als auch an der Wahl der Forschungsgegenstände selbst, die auf den ersten Blick von sehr peripherer Bedeutung sein können (wie z. B. ein einfacher Handwerker oder Soldaten aus den Mannschaftsdienstgraden).

Dieses Vorgehen ist nicht zuletzt darin begründet, dass die Historikerinnen und Historiker sich offensiver zu ihrer Subjektivität bekennen und sie stärker in die eigene Arbeit integrieren. Das geht mitunter soweit, dass Einzelne die Geschichte der eigenen Familie oder Verwandtschaft zum (wissenschaftlichen) Thema machen (etwa Philippe Artières oder Ivan Jablonka). Ebenso wird der literarischen Dimension der historischen Texte mehr Aufmerksamkeit geschenkt, bis hin zu Reflexionen über den fiktionalen Charakter der Geschichtsschreibung und ihren Bezügen zum literarischen Feld ${ }^{7}$. Diese Veränderungen werden im Übrigen - während sie noch im Geschehen begriffen sind von den Historikerinnen und Historikern selbst untersucht und analysiert, weshalb sich 
in letzter Zeit die kritischen Bilanzen und Forschungsüberblicke erheblich vermehrt haben (siehe dazu die Bibliographie am Ende des Beitrags).

\section{a) Small is beautiful}

13 Während in den 1970er und 1980er Jahren die markanten geschichtswissenschaftlichen Dissertationen und Veröffentlichungen oft eine Region oder eine wichtige Stadt zum Thema hatten und einen relativ langen Zeitraum untersuchten (mitunter zwei oder drei Jahrhunderte), stehen heute erheblich eingeschränktere Themen im Fokus der historischen Zunft. Dies liegt nicht in erster Linie an den Arbeitsbedingungen, die es notwendig machen, die Ambitionen zu zügeln, sondern vor allem an der Auffassung, dass ein sorgfältig bearbeiteter Forschungsgegenstand Ergebnisse hervorbringt, die in ihrer Bedeutung nicht einfach von der Weite des untersuchten Rahmens abhängig sind. Als Einfluss ist hier natürlich die Mikrogeschichte (vor allem italienischen Ursprungs) zu nennen, die in den 70er Jahren schon sehr dynamisch war. Doch bei weitem nicht alle Arbeiten mit einem eng gefassten Untersuchungsgegenstand fallen in diese Kategorie die Mikrogeschichte bleibt eine Herangehensweise unter vielen.

Eine solche Eingrenzung der Perspektive äußert sich in verschiedenen Formen und zeugt von unterschiedlichen methodologischen Ambitionen. Eine erste Version des "small is beautiful«besteht darin, sich auf ein Dokument oder eine Serie von Quellen als eigenständigen historischen Gegenstand $\mathrm{zu}$ konzentrieren - dieser Ansatz wird mitunter »neue Gelehrsamkeit«(nouvelle érudition) genannt. So untersuchen beispielsweise manche Mediävisten ein Kopialbuch oder eine Urkundensammlung in allen seinen bzw. ihren Aspekten als ein Objekt an sich und nicht nur, um eine Sozial- oder eine Wirtschaftsgeschichte zu schreiben (etwa Patrice Beck am Beispiel der burgundischen Herdsteuerlisten) ${ }^{8}$. Auf eine andere Weise kann eine archivalische Quelle aber auch als Grundlage für eine experimentelle Geschichte dienen, wenn mehrere Historikerinnen und Historiker ihre Analyse der gleichen Akte liefern - und umso mehr, wenn diese Akte als Zufallsfund auf einem Flohmarkt erstanden wurde (wie im Fall eines lokalen Bankiers des 19. Jahrhunderts, Daniel Bertrand). In Le Dossier Bertrand. Jeux d'histoire (2008) hat sich eine Gruppe von Historikerinnen und Historikern also mit einem Gegenstand beschäftigt, dessen Auswahl zu Beginn keinerlei wissenschaftlichen Kriterien unterlag. Das Buch fügt die verschiedenen Fragmente der beteiligten Personen nach Art einer Collage zusammen und folgt dabei einer "Spielregel«. Einen besonderen Akzent setzen im Buch stilvolle Photographien, die aus der Akte stammen.

Es kann aber auch darum gehen, eine bereits gut erforschte Periode oder ein Ereignis aus dokumentarischer Perspektive neu $\mathrm{zu}$ beleuchten. Ein solcher "philologischer Ansatz« wurde etwa von Pierre Gervais, Pauline Peretz und Pierre Stutin für die DreyfusAffäre verfolgt, wobei hier von einer Rekonstruktion der »Geheimakte« und ihren möglichen »Versionen« aus neue Blickwinkel auf das Ereignis erschlossen wurden und damit die Debatte neu belebt wurde ${ }^{9}$. Auch die Kulturgeschichte findet im eng umgrenzten Gegenstand (insbesondere literarische oder künstlerische Kreationen) einen leichten Zugang, um über die Zirkulation von Wissensbeständen oder die Geschichte von Texten nachzudenken, wie es kürzlich Roger Chartier am Beispiel eines Stücks von Shakespeare ( „Cardenio«) bewiesen hat, dessen Manuskript verschollen ist ${ }^{10}$.

16 Wie Alain Dewerpes großes Buch (2006) über die Demonstration von Charonne am 8. Februar 1962 zeigt, eignen sich bestimmte Ereignisse besonders gut, um eigentlich 
begrenzte Ereignisse einer tiefergehenden historischen Analyse zu unterziehen. Der Rahmen - eine einzige (freilich sehr wichtige) Demonstration - ist hier scheinbar sehr eng gezogen, wenn man bedenkt, dass es auch möglich gewesen wäre, von der Geschichte der Polizei während des Algerienkriegs oder von allen Demonstrationen dieser Epoche auszugehen. Die Bearbeitung des Themas jedoch ist gewaltig: Es werden nicht nur minutiös die Ereignisse rekonstruiert und die Frage nach der Verantwortung der Tragödie am Ende der Demonstration gestellt (die Polizei schoss auf die Demonstranten und tötete mehrere von ihnen), sondern es werden ebenso die Gewaltausübung eines sich im Kriegszustand befindenden Staates und die politischen Implikationen des Verhaltens und der Praktiken der Polizei aufgezeigt. Aus einer anderen Perspektive hat Didier Lett einen einzigen Kanonisierungsprozess, den von Nikolaus von Tolentino am Anfang des 14. Jahrhunderts, als Ausgangspunkt genommen, um eine Analyse der schriftlichen Überlieferung und eine Sozialgeschichte der »Prozess-Gesellschaft « zu schreiben ${ }^{11}$.

So werden Nebendarsteller oder gar Komparsen der Geschichte zu den neuen Helden der historischen Zunft. Ein Wegbereiter dieser Herangehensweise war Alain Corbin, der, weitgehend zufällig und ausgehend von einer dünnen Quellenlage, das Leben und die Lebenshorizonte von Louis-François Pinagot rekonstruierte, eines im 19. Jahrhundert in der Region Perche lebenden armen Holzschuhmachers. Die Historiker Fabrice Virgili und Danièle Voldman wiederum erzählen in "La garçonne et l'assassin. Histoire de Louise et de Paul, déserteur travesti, dans le Paris des années folles « (2011) die außergewöhnliche Biographie von Paul Grappe und seiner Partnerin Louise, die ihn schließlich ermordete. Deserteur im Ersten Weltkrieg, versteckte sich Grappe, indem er sich travestierte, er war bisexuell, „Swinger«, gelegentlich auch Zuhälter und wurde immer mehr zum Alkoholiker. Diese Geschichte ist weit davon entfernt, eine bloße Anekdote zu sein, vielmehr gleicht sie einer Sozial-, Geschlechter- und Erinnerungsgeschichte des Ersten Weltkriegs. Gérard Noiriel hat seine Arbeiten zu Immigration, Rassismus und Identität fortgesetzt, indem er das Leben des Clowns Chocolat nachzeichnete, eines schwarzen Bühnenkünstlers, der 1917 starb $^{12}$. Ivan Jablonka schließlich hat das Leben seiner kommunistischen und jüdischen Großeltern rekonstruiert, die unter den Nazis verfolgt und schließlich deportiert wurden ${ }^{13}$.

Begrenzte Untersuchungsfelder eignen sich vielleicht leichter für Experimente, Versuche und »fragile Begegnungen« als große Gegenstände. So hat Gérard Noiriel mit »Chocolat« die Berufe des Historikers und des Theaterschaffenden verbunden, indem er beratend ein Theaterstück zum gleichen Thema begleitete. Diese Praxis hat er durch weitere Bühnenerfahrungen fortgesetzt, z. B. bei einem Stück über Marie Curie. Als Beleg ließen sich aber auch das Dossier Bertrand oder die Arbeit eines Historikers (Laurent Feller), einer Anthropologin (Florence Weber) und einer Wirtschaftswissenschaftlerin ( Agnès Gramain) zur Preisbildung und zum Grundstückshandel im Hochmittelalter anführen, die vor allem der Frage von "Zusammenhängen von interpersonalen Beziehungen und Handelstransaktionen « nachgeht ${ }^{14}$. Auf der Basis der KopialbuchChronik von San Clemente a Casauria trugen sie alle Informationen zusammen, die Karol, den Sohn des Liutprand, und den Marktflecken Vico Teatino betreffen: 101 überaus gehaltvolle Dokumente, die über die Aktivitäten eines zu einer Art Bauernelite gehörenden wichtigen Zwischenhändlers Aufschluss geben.

19 Dieser große (bzw. kleine) Beobachtungsmaßstab schließt dabei nicht unbedingt die Verwendung von quantitativen Daten und Statistiken aus, wie die Arbeit von Claire Zalc und Nicolas Mariot über die Juden von Lens während der Besatzungszeit zeigt (2010) ${ }^{15}$. 


\section{b) "Large« is beautiful}

\section{Beziehungen und Zusammenhänge zwischen ungleichen Orten und Welten hervorhebt)} privilegiert Arbeiten mit einem weiten Fokus, welche die Beziehungen und Interaktionen zwischen Regionen und Nationen hervorheben. Auf diesem Gebiet waren französische Historikerinnen und Historiker sicher keine treibenden Kräfte, aber sie haben sich diesen Ansatz der Geschichtsschreibung auf ihre eigene Art angeeignet. Sie taten dies zunächst, indem sie weltgeschichtliche Fragestellungen auf Epochen anwandten, die bislang kaum oder wenig unter diesem Blickwinkel betrachtet wurden, wie z.B. das Mittelalter. "L'histoire du Monde au Xve siècle«, herausgegeben von Patrick Boucheron (2009), versammelt eine Vielzahl von Historikerinnen und Historikern, die sich mit den Herausforderungen einer Welt-Geschichte auseinandersetzen. Spezifische Fragestellungen werden auch in Seminaren behandelt, etwa dem von Chloé Maurel (École Normale Supérieure, Rue d'Ulm) oder dem von Stéphane van Damme und Romain Bertrand (Institut d'Études Politiques de Paris) über die Konstruktion und die Zirkulation von Wissensbeständen beim Aufeinandertreffen von Weltreichen (»rencontre impériale«).

In "L'Histoire à parts égales. Récits d'une rencontre Orient-Occident, XVI-XVII ${ }^{\mathrm{e}}$ siècle« (2011) versucht er, eine »symmetrische« , d.h. "gleichberechtigte« Geschichte des Zusammentreffens von Holländern, Malaien und Einwohnern Javas zwischen Ende des 16. und Anfang des 17.Jahrhunderts zu schreiben. Voraussetzung eines solchen Vorhabens ist es, Quellen von beiden Seiten des Zusammentreffens zu benutzen und dabei jeder ihre Besonderheiten und Weltsichten $\mathrm{zu}$ lassen, also nicht überall »Gemeinsamkeiten« zu sehen. Ebenso gehört dazu, zu erkunden, wie jede Seite sich an der anderen maß - und zwar mit den jeweils eigenen Kriterien (mitunter war es nur Gleichgültigkeit) - und den Akteuren die Wahrnehmung ihres Zusammentreffens selbst zu überlassen. Aufgezeigt wird von einem solchen Vorgehen auch, dass solche fernen Bevölkerungen weitaus mehr Kontakte hatten als nur die Europäer, die nur eine Beziehung zwischen anderen darstellten.

23 Es ist noch zu früh, um den Einfluss von Global- und Weltgeschichte bzw. connected history auf die französische Geschichtsschreibung zu bestimmen, denn originäre Forschungsarbeiten zu diesem Thema, wie die von Romain Bertrand, sind noch nicht sehr zahlreich. Sicher ist jedoch, dass diese Ansätze bereits einen wichtigen Platz in den Diskussionen einnehmen, wie zahlreiche Artikel und Themenausgaben von Zeitschriften zeigen.

Meist auf implizite Weise, manchmal aber auch offensichtlicher, scheint die Globalgeschichte in Frankreich die gute alte Nationalgeschichte in Frage zu stellen. In der Tat zeigt sie zahlreiche alternative Erzählungen zur Nationenbildung auf, der ein guter Teil der französischen Historiographie zuneigt. Dieser Gegensatz wurde anlässlich des Historikertreffens in Blois 2011 besonders deutlich, dessen zentrales Thema »Der 
Orient « war. Zur gleichen Zeit wurde auch das Projekt einer »Maison de l'histoire de France« (angestoßen vom damaligen Präsidenten Nicolas Sarkozy) diskutiert und setzte die Debatten fort, die bereits im ersten Teil des Artikels erwähnt wurden. Gaïdz Minassian hat es in einem Bericht für Le Monde auf den Punkt gebracht, der es verdient, ausführlich zitiert zu werden. Seine Analyse ist darüber hinaus interessant, weil sie einen Blick von außen auf die Historikerzunft wirft.

Jene historische Konfiguration, die die Welt um die europäische oder westliche Gesellschaft herum organisierte, welche wiederum als ein im Vergleich zu den anderen dominierendes ,Wir' definiert war, ist aus unserer globalisierten Welt verschwunden. Das ,Wir' ist nicht mehr westlich, es wird global. Und im Zuge dieser Entgrenzung verliert die westliche Welt das Monopol der Geschichtsschreibung und sein Erbe wird durch die Rückkehr des Verdrängten in Frage gestellt. Aus der Dezentrierung des globalen Raums ist eine historiographische Konfrontation hervorgegangen, bei der sich Verteidiger der Nationalgeschichte, wie das Akademiemitglied Pierre Nora, und Befürworter einer globalen oder Beziehungs-Geschichte, wie der indische Forscher Sanjay Subrahmanyam, gegenüberstehen, dessen Vorträge in den vergangenen vier Tagen sehr viel Beachtung gefunden haben.

Und hier zeigt sich ein drittes Paradox : Dem Historikertreffen in Blois, wo der nationalgeschichtliche Ansatz vorherrschend bleibt, kommt das Verdienst zu, die epistemologische Bedeutung der Globalgeschichte bestätigt zu haben, während Frankreich in seinen institutionellen, universitären, schulischen, sprachwissenschaftlichen und verlegerischen Bereichen diese neue historiographische Tendenz noch kaum in seine Arme geschlossen hat. [...] Zwar eröffnete Sanjay Subrahmanyam die 14. Ausgabe des Treffens von Blois, doch war es nicht vielleicht doch Pierre Nora, der mit seinem Abschlussvortrag das letzte Wort hatte ? Der Erste sprach sich als Mittler zwischen Orient und Okzident für die Gleichberechtigung der Wissenssysteme aus und warb für Koproduktion und Verständigung gegen die Logik der Dominanz. Zwar wird die Geschichte seit mehr als 200 Jahren von den westlichen Eroberern geschrieben, doch »Der Sieger bleibt nicht immer Sieger« , so der indische Historiker, denn das Rad dreht sich weiter ${ }^{16}$.

Sicher ist es möglich $\mathrm{zu}$ argumentieren, dass sich die verschiedenen Beobachtungsmaßstäbe nicht notwendigerweise widersprechen müssen, sondern als zwar verschiedene, aber komplementäre Ansätze betrachtet werden können. Dennoch vermischen sich die Entscheidungen von Historikerinnen und Historikern hier leicht mit den Aspekten, die Minassian in den Vordergrund gestellt hat. Das heißt, dass bestimmte Diskurse über und bestimmte Praktiken der Geschichte Frankreichs sich auch einer nostalgischen Form des Engagements verschrieben haben, während die Wahl von bestimmten Untersuchungsgegenständen der connected history oder der Globalgeschichte Position bezieht für eine Öffnung hin zur Alterität in einem politischen Umfeld, das von neuen Formen des Fremdenhasses geprägt ist.

\section{Tendenzen}

In dieser kurzen Übersicht kann keine umfassende Bestandsaufnahme der verschiedenen Forschungsfelder und neuen Objekte bzw. Bereiche geleistet werden. In diesem dritten Abschnitt sollen dennoch einige Tendenzen der französischen Geschichtsschreibung herausgestellt werden, die neue Perspektiven öffnen oder alte Wege neu ausschreiten. 
Dabei soll nicht vergessen werden, dass ein derartiges Inventar keine Repräsentativität beanspruchen kann. Der Grund hierfür ist, dass die neuesten und innovativsten Tendenzen oft nur wenige Personen betreffen oder zumindest Bereiche des Feldes, die ihre eigenen Charakteristika aufweisen, z.B. an einem Ort oder in einer Institution lokalisiert sind. Eine Gesamtübersicht sähe deshalb anders aus - sie müsste auch klassische und bekannte Arbeiten einbeziehen wie Schlachtenbeschreibungen, narrative Biographien oder regionale Monographien. Auch trägt die Art und Weise, wie die einzelnen Richtungen hier präsentiert werden, dazu bei, Vorgehensweisen $\mathrm{zu}$ formalisieren oder zu verdinglichen, die in Wirklichkeit viel flexibler sind oder sich kaum so einfach fassen lassen, wie die gewählten Bezeichnungen es vermuten lassen. Schließlich handelt es sich natürlich um eine subjektive Auswahl.

Eine erste Vorgehensweise besteht darin, die großen Zuschnitte der Disziplin (Politikgeschichte, Wirtschaftsgeschichte, Sozialgeschichte usw.) in den Blick zu nehmen und festzustellen, wie stark sich das Feld der sogenannten Kulturgeschichte seit einigen Jahrzehnten entwickelt hat und wie vielfältig es geworden ist. Dabei müsste natürlich der Begriff der „Kultur" selbst hinterfragt werden - der Einfachheit halber verwenden wir ihn hier im für die Geschichtsschreibung üblichen Sinn. Auffällig wird dann die erhebliche Erweiterung der Forschungsgegenstände, ob nun Weltanschauungen und/oder Praktiken untersucht werden bzw. vom Körper (Sport, Schmuck, Bräunung) ${ }^{17}$ und von Sexualität oder von Arten und Weisen des Lesens oder Essens die Rede ist. Nicht ganz ohne Grund wurde ein großer und stark sichtbarer Teil der Kulturgeschichte dafür kritisiert, die Repräsentationen, die vorherrschenden Bilder und die am leichtesten zugänglichen Medien zu privilegieren - auf Kosten einer Analyse von Machtstrukturen und sozialen Interaktionen. Es gibt jedoch - Historiker wie Roger Chartier oder Daniel Roche haben darauf seit Langem hingewiesen - eine Sozialgeschichte der Kultur, die von ganz anderer Intensität ist und beispielsweise in der Geschichte des Buches oder der Geschichte des Konsums deutlich wird. Das letzte Thema wird vielfältig fortgesetzt und berührte dabei auch die Politik- und die Geschlechtergeschichte ${ }^{18}$.

Auch die Arbeiten Antoine Liltis zu Salon und Renommee vereinen historische Sorgfalt und soziologische Überlegungen. Stéphane van Damme erneuert, in einem lebhaften Dialog mit den Sozialwissenschaften und der Wissenssoziologie, die Erforschung des Wissens und seiner Verankerung in weiten Netzwerken. Eines seiner letzten Werke, »Métropoles de Papier« (2012), befasst sich mit der Rolle von Gelehrten, seien sie Historiker oder Geologen, bei der Definition und Legitimation der Städte vom 17. bis zum 19. Jahrhundert. In eben diesem Feld der Wissensgeschichte brachten die letzten Jahre vor allem wichtige Arbeiten zum Thema der Kolonien und zu den kolonialen Situationen hervor, sei es im Bereich des Rechts, der »Kolonialwissenschaften«oder des Bildungswesens (Pascale Barthélemy, Sophie Eckert-Dulucq, Emmanuelle Saada, Emmanuelle Sibeud, Pierre Singaravélou, Pierre Vermeren).

Eine zweite Herangehensweise besteht darin, die Bereiche zu identifizieren, die sich im Wachstum befinden, jedoch noch nicht unter die oben genannten großen Forschungsrichtungen fallen. Ohne Zweifel ist so die Geschlechtergeschichte inzwischen auch in Frankreich, trotz der Verspätung der angelsächsischen Welt gegenüber, ein von vielen praktizierter Forschungsgegenstand geworden. Dies zeigt sich an Arbeiten von Historikerinnen und Historikern verschiedener Epochen (wie z. B. Didier Lett für das Mittelalter), an ihrer Institutionalisierung (durch eigene Lehrveranstaltungen) und auch durch Arbeiten, deren primärer Ansatz sie nicht ist (wie z.B. im Fall von Marie- 
Emmanuelle Chessel in ihren Arbeiten zum Konsum und $\mathrm{zu}$ den Konsumentengemeinschaften am Anfang des 20. Jahrhunderts, 2012) ${ }^{19}$. Der geschlechtergeschichtliche Ansatz hat daneben dazu beigetragen, die Arbeiten zur Konstruktion der Maskulinitäten, zu unscharfen Identitäten und zu allen möglichen Formen der Sexualität zu vervielfältigen. Erwähnt werden soll auch die bedeutende Entwicklung der Umweltgeschichte, zwar auch hier mit Verspätung gegenüber der angelsächsischen Welt, jedoch mit einer spezifisch französischen Ausprägung (vor allem auf institutioneller Ebene), die im Zusammenhang mit einer Geschichte der Tiere steht (angewandt unter verschiedenen Fragestellungen von Eric Baratay, Jean-Marc Moriceau und Michel Pastoureau) ${ }^{20}$.

30 In beiden Fällen geht es darum, nicht-menschlichen Objekten (Landschaft, Tiere) neue Konsistenz zu geben, sei es an und für sich, mit einer eigenen Geschichtlichkeit, sei es in ihrer Verbindung zum Menschen, wie es die Wissenschaftssoziologie und die "pragmatische Soziologie« (Luc Boltanski u. a.) bereits ausführlich im Hinblick auf Gegenstände getan haben. Diese Fragestellungen führen zur Untersuchung von verschiedenen Bereichen wie etwa kolonialen oder maritimen Räumen; sie ziehen aber auch ein Nachdenken über neue historische Zeiteinteilungen nach sich (wie z. B. das Anthropozän, eine neue geologische Ära, die durch die Umwälzung der Gleichgewichte geprägt ist, wie sie aus den menschlichen Aktivitäten im Gefolge der industriellen Revolution resultierte - siehe dazu die Arbeiten von Jean-Baptiste Fressoz und Christophe Bonneuil) 21 .

31 Schließlich können drittens Themen in den Blick genommen werden, die in den letzten Jahren besonders fruchtbare Arbeiten hervorgebracht haben. Dazu zählen beispielsweise Studien zum Begriff der öffentlichen Ordnung und - im Zusammenhang mit ihm, durchaus aber auch unabhängig davon - zum Begriff der Gewalt. In diesem Bereich hat die französische Geschichtswissenschaft eigene Akzente gesetzt, etwa bei einer Neubestimmung des Verhältnisses von Gehorsam und Ungehorsam im Krieg (André Loez zu den Meutereien von 1917, 2010), bei der Geschichte der Ordnungskräfte, der Polizei und der Gendarmerie in all ihren Facetten: im Einsatz, in der Interaktion oder als Berufszweig (Christian Chevandier, Vincent Denis, Quentin Deluermoz und Aurélien Lignereux ...). Der Begriff der Gewalt wird in einem von Patrick Boucheron und Vincent Azoulay herausgegebenem Sammelband auch für das intellektuelle Feld untersucht ${ }^{22}$.

Die Geschichte der Emotionen schließlich befasst sich mit allen Epochen und bringt sehr ergiebige Neuinterpretationen von klassischen Fragestellungen hervor. Mitunter verbindet sie sich mit dem neuen Interesse für Geräusche und Geräuschlandschaften, das hinter dem "acoustic turn« in den Sozialwissenschaften steht. Auch hier durchmessen Arbeiten alle Epochen der Geschichte und öffnen sich verschiedenen Untersuchungsbereichen, wovon u.a. eine von Pierre Prétou und Frédéric Chauvaud organisierte Tagung zum Verhältnis von öffentlichem »Geschrei« und der Aktivität des Justizapparats zeugt, die zehn Jahre nach einem ersten Sammelband zur Geschichte des Rufens und Schreiens stattfand ${ }^{23}$. In diesem Zusammenhang entwerfen Historiker auch neue Perspektiven für die Sozial- und Politikgeschichte, mit Arbeiten zu den Begriffen von Ehre und Unehre oder Schande etwa (Claude Gauvard und Antoine Destemberg für das Mittelalter und Anne Simonin für die neuere und neueste Geschichte).

33 Am Ende dieses kurzen Überblicks ist die Auflösung der Beobachtungsmaßstäbe, der Forschungsgegenstände und der Ansätze evident; manche sorgen sich sogar über das Fehlen übergreifender Ansätze in der französischen Geschichtsschreibung. Doch diese 
Fragmentierung ist auch eine Quelle von Kreativität. Das Ausloten der Verbindungen zwischen Geschichte und Literatur wird flexibler und gewinnt an historischer Tiefenschärfe, die kontrafaktische Geschichte wird noch einmal aufgerollt, engagierte Formen von Geschichtsschreibung gewinnen an Robustheit und experimentelle Arten der Historiographie werden durch die Reflexion bereichert. Ob diese Tendenzen ein gleichwertiges Echo auch in den universitären Instanzen finden, ist hingegen nicht sicher - aber das steht auf einem anderen Blatt.

Blanc, William, Chéry, Aurore, Naudin, Christophe, Les Historiens de garde. De Lorànt Deutsch à Patrick Buisson, la résurgence du roman national, Paris 2013.

Boucheron, Patrick, Faire profession d'historien, Paris 2010.

Brilli, Elisa, Dufal, Blaise, Dittmar, Pierre-Olivier (Hg.), Faire l'anthropologie historique du Moyen Âge, in : L'Atelier du Centre de Recherches Historiques, juin 2010, http:// acrh.revues.org/1911.

Carrard, Philippe, Le Passé mis en texte. Poétique de l'historiographie française contemporaine, Paris 2013.

Delacroix, Christian, Dosse, François, Garcia, Patrick, Offenstadt, Nicolas (Hg.),

Historiographies. Concepts et débats, 2 Bde., Paris 2010.

Delacroix, Christian, Dosse, François, Garcia, Patrick, Les courants historiques en France, XIX-XXe siècle, Paris 2007 [erste Auflage 1999].

Dumoulin, Olivier, Le rôle social de l'historien. De la chaire au prétoire, Paris 2003.

Garcia, Patrick, Leduc, Jean, L'enseignement de l'histoire en France. De l'Ancien Régime à nos jours, Paris 2003.

Granger, Christophe (Hg.), À quoi pensent les historiens ? Faire de l'histoire au XXI siècle, Paris 2013.

Offenstadt, Nicolas, L'Historiographie, Paris 2011.

Offenstadt, Nicolas, L'histoire, un combat au présent, Paris 2014.

Sirinelli, Jean-François, Cauchy, Pascal, Gauvard, Claude (Hg.), Les historiens français à

l'œuvre, 1995-2010, Paris 2010.

\section{NOTES}

1. Vgl. Nicolas Offenstadt, L'histoire bling-bling, Paris 2009; William Blanc, Aurore Chéry, Christophe Naudin, Les Historiens de garde. De Lorànt Deutsch à Patrick Buisson. La résurgence du roman national, Paris 2013. Zur deutschen Debatte und den daraus resultierenden Fragen für die Geschichtswissenschaft vgl. beispielsweise: Gangolf Hübinger, Über die Aufgaben des Historikers, Berlin 2012; Peter Steinbach, Geschichte im politischen Kampf. Wie historische Argumente die öffentliche Meinung manipulieren, Bonn 2012 und Wolfgang Hardtwig, Verlust der Geschichte oder wie unterhaltsam ist die Vergangenheit?, Berlin 2010.

2. Vgl. hierzu und zum Folgenden: Claude Liauzu, Gilles Manceron (Hg.), La colonisation, la loi, l'histoire, Paris 2006; Romain Bertrand, Mémoires d'Empire, Bellecombe-en-Bauges 2006; Gerard Noiriel, Nicolas Offenstadt, Histoire et politique. Autour d'un débat et de certains usages, in: Nouvelles fondations 2 (2006), S.65-75 (http://www.cairn.info/revue-nouvellesfondations-2006-2-page-65.htm. Alle Links zuletzt eingesehen am 14.7.2014. 
3. Patrick Garcia, France 2005, une »crise historique en perspective«, in: Bogumil Jewsiewicki, Érika Nimis (Hg.), Expériences et mémoire. Partager en français la diversité du monde, Paris 2008, S. 337-352.

4. Vgl. insbesondere das sogenannte "Gesetz Gayssot" vom 13. Juli 1990 gegen den Negationismus, das den Tatbestand der »Infrage-Stellung eines Verbrechens gegen die Menschlichkeit« schafft, das Gesetz vom 29. Januar 2001 über die Anerkennung des Genozids in Armenien oder das sogenannte "Gesetz Taubira« vom 21. Mai 2001 über den Sklavenhandel und die Sklaverei, die als Verbrechen gegen die Menschlichkeit anerkannt wird. Das Gesetz fordert, dass in den Lehrplänen »der Sklavenhandel und die Versklavung den wichtigen Platz einnehmen soll, der ihnen gebührt«. Vgl. dazu: Boris Adjemian, Le débat inachevé des historiens français sur les "lois mémorielles« et la pénalisation du négationnisme: retour sur une décennie de controverse, in: Revue arménienne, 15. décembre 2012, S. 9-34 [In Frankreich werden Gesetze regelmäßig nach den Personen - Minister, Abgeordnete usw. - benannt, die sie auf den Weg gebracht haben. Anm. d. Ü.].

5. Patrick Boucheron, Faire profession d'historien, Paris 2010, S. 136 und vor allem Valentin Groebner, Das Mittelalter hört nicht auf. Über historisches Erzählen, München 2008.

6. Vgl. die neuere Gesamtdarstellung von Pierre Singaravélou (Hg.), Les Empires coloniaux, $\mathrm{XIX}^{\mathrm{e}}$ $\mathrm{xx}^{\mathrm{e}}$ siècle, Paris 2013.

7. Vgl. zu diesen Fragen Judith Lyon-Caen, Dinah Ribard, L'Historien et la littérature, Paris 2010, und das Themenheft von »Le Débat«,165, Mai-août 2011: »L'histoire saisie par la fiction«.

8. Patrice Beck, Archéologie d'un document d'archives. Approche codicologique et diplomatique des cherches des feux bourguignonnes (1285-1543), préface d'Olivier Guyotjeannin, Paris 2006 (Études et rencontres de l'École des chartes, 20).

9. Pierre Gervais, Pauline Peretz, Pierre Stutin, Le dossier secret de l'affaire Dreyfus, Paris 2012.

10. Roger Chartier, Cardenio entre Cervantes et Shakespeare. Histoire d'une pièce perdue, Paris 2011. Ein erster, etwas traditioneller Überblick zur Kulturgeschichte bei Philippe Poirrier, Les enjeux de l'histoire culturelle, Paris 2004. Vgl. auch Olivier Dumoulin, Esquisse d'un bilan de l'histoire culturelle en France depuis 1995, in: Jean-François Sirinelli, Pascal Cauchy, Claude Gauvard (Hg.), Les historiens français à l'œuvre, 1995-2010, Paris 2010, S. 237-259.

11. Didier Lett, Un procès de canonisation au Moyen Âge. Essai d'histoire sociale. Nicolas de Tolentino, 1325, Paris 2008.

12. Gerard Noiriel, Chocolat, clown nègre: l'histoire oubliée du premier artiste noir de la scène française, Montrouge 2012.

13. Ich selbst habe über die Bedeutung des öffentlichen Ausrufens und die Möglichkeitsbedingungen eines öffentlichen Raumes im Mittelalter gearbeitet und versucht, die Erfahrungen eines Stadtschreiers im 15. Jahrhundert zu rekonstruieren und nachzuverfolgen: Nicolas Offenstadt, En place publique. Jean de Gascogne, crieur au Xve siècle, Paris 2013.

14. Laurent Feller, Agnès Gramain, Florence Weber, La Fortune de Karol. Marché de la terre et liens personnels dans les Abruzzes au Haut Moyen Âge, Rom 2005.

15. Nicolas Mariot, Claire Zalc, Face à la persécution: 991 Juifs dans la guerre, Paris 2010 (Fondation pour la mémoire de la Shoah).

16. Gaïdz Minassian, L'histoire globale peine encore à supplanter le »roman national« en France, in: Le Monde, 19.10.2011.

17. Vgl. dazu im weiteren Kontext, "Le Corps, territoire politique«, Cahiers d'histoire revue d'histoire critique, 118, janvier-mars 2012.

18. Vgl. zum Beispiel die Festschrift für Daniel Roche: Vincent Millot, Philippe Minard, Michel Porret (Hg.), La Grande chevauchée. Faire de l'histoire avec Daniel Roche, Genf 2011.

19. Siehe im Allgemeinen die Zeitschrift : Clio. Femmes, genre, histoire. 
20. Vgl. beispielsweise: Éric Baratay, Le point de vue animal: une autre version de l'histoire, Paris 2012; Jean-Marc Moriceau, L'homme contre le loup: une guerre de deux mille ans, Paris 2011; Michel Pastoureau, L'ours: histoire d'un roi déchu, Paris 2007.

21. Christophe Bonneuil, Jean-Baptiste Fressoz, L'événement anthropocène. La Terre, l'histoire et nous, Paris 2013.

22. Patrick Boucheron, Vincent Azoulay (Hg.), Le mot qui tue. Les violences intellectuelles de l'Antiquité à nos jours, Seyssel 2009.

23. Didier Lett, Nicolas Offenstadt, Haro! Noël! Oyé! Pratiques du cri au Moyen Âge, Paris 2003. Frédéric Chauvaud, Pierre Prétou (Hg.), Clameur publique et émotions judiciaires de l'Antiquité à nos jours, Rennes 2013.

\section{RÉSUMÉS}

Der Text skizziert einige Grundtendenzen der französischen Geschichtsschreibung, die sich in den letzten zwanzig Jahren entwickelt haben. Zunächst wird die Geschichtswissenschaft als Disziplin ins Verhältnis zu jenen öffentlichen Debatten gesetzt, die Einfluss auf die akademische Welt ausüben. Anschließend wird auf die unterschiedlichen Maßstabsebenen eingegangen, mit denen französische Historikerinnen und Historiker bei ihrer Arbeit heute operieren, sowie auf die Öffnung bei der Definition neuer Forschungsthemen. Schließlich werden einige besonders ertragreiche Forschungsfelder vorgestellt.

\section{INDEX}

Schlüsselwörter : Frankreich, Historiographie, öffentliche Nutzung von Geschichte, Geschichtsschreibung, Geschichte, Politik, Kollektives Gedächtnis

\section{AUTEURS}

\section{NICOLAS OFFENSTADT}

PD Dr. Nicolas Offenstadt ist Maître de Conférences an der Universität Paris I PanthéonSorbonne.

Mail: Nicolas.offenstadt[at]univ-paris1.fr 